\title{
Parasites, vecteurs de pathogènes et changements climatiques
}

\author{
Parasites, vectors and global changes
}

\author{
Gérard Duvallet \\ UMR 5175, Centre d'Écologie Fonctionnelle et Évolutive, Université Paul Valéry, Montpellier 3, \\ 34199 Montpellier Cedex 5, France \\ gerard.duvallet@univ-montp3.fr
}

Résumé - Des modifications dans la distribution et/ou le comportement de nombreuses espèces vivantes sont observées par les biologistes. Ces derniers tentent d'analyser la part du réchauffement climatique actuel dans ces modifications. L'influence du changement climatique sur le risque d'émergence de maladies vectorielles, en particulier, est devenu un thème préoccupant pour nos sociétés. Certains arthropodes représentent un danger pour la santé, parce qu'ils sont simplement transporteurs de pathogènes, ou qu'ils sont hématophages. Dans ce dernier cas, en plus de la prédation sanguine, ils peuvent être vecteurs biologiques ou mécaniques d'agents pathogènes. Les maladies induites sont connues essentiellement dans les zones inter-tropicales, mais certaines d'entre elles ont déjà fait des incursions dans les régions tempérées. On peut supposer que le réchauffement climatique aura des répercussions sur la distribution latitudinale et altitudinale des vecteurs, mais aussi sur leurs périodes d'activité au cours de l'année, leur longévité et leur densité, et aussi sur la durée d'incubation des agents pathogènes. Sont envisagés comme exemples le cas du moustique Aedes albopictus, qui s'est récemment implanté en France, le cas des phlébotomes, vecteurs de leishmaniose chez le chien et chez l'homme en région méditerranéenne, la récente épidémie à virus West Nile aux USA et en France, celui des Culicoïdes, vecteurs de la fièvre catarrhale du mouton en Corse et qui menace le continent, enfin celui des maladies transmises par tiques.

Mots clés - maladies vectorielles, changement climatique, arthropodes, parasites

\begin{abstract}
Modifications in the distribution and/or the behavior of many living species are observed by the biologists. The latter try to analyze the importance of the global warming in these modifications. The influence of the climatic change on the risk of emergence of vectorial diseases, in particular, became an alarming topic for people. Some arthropods represent a danger to health, because they are simply conveyors of pathogens, or that they are hematophagous. In this last case, in addition to the blood predation, they can be biological or mechanical vectors of pathogenic agents. The induced diseases are known primarily in the intertropical zones, but some of them already made incursions into the temperate areas. One can suppose that the global warming will have effects on the latitudinal and altitudinal distribution of the vectors, but also over their phenology during the year, their longevity and their density, and also over the period of incubation of the pathogenic agents. Are considered like examples the case of the mosquito Aedes albopictus, which was
\end{abstract}


recently established in France, the case of the sandflies, vectors of leishmaniosis in the dog and the man in Mediterranean area, the recent epidemic with West Nile virus in the USA and in France, that of Culicoïdes, vectors of the blue tongue disease of the sheep in Corsica and which threatens the continent, finally that of the tick-transmitted diseases.

Key words - vectorial diseases, global warming, arthropods, parasites

L'apparition du virus West Nile transmis par moustiques sur le continent américain pour la première fois en 1999 à New York, sa réapparition en France en Petite Camargue en 2000 après plus de 30 ans de silence épidémiologique, l'épidémie de fièvre catarrhale du mouton apparue en Corse en 2000 sont autant d'exemples qui ont attiré l'attention du public, des médias et des responsables sur les maladies émergentes ou ré-émergentes transmises par vecteurs (Epstein, 2001). Parmi les explications possibles à ces phénomènes épidémiologiques, les biologistes tentent de faire la part des modifications des écosystèmes, du rôle grandissant des transports, des échanges intercontinentaux et des changements climatiques.

\section{C'est un thème d'actualité...}

En écologie, 3 grands domaines de recherche sont reconnus désormais comme prioritaires au niveau international :

- l'étude de la biodiversité;

- l'étude des changements globaux, qu'il s'agisse des changements climatiques ou des atteintes à la biodiversité ;

- le développement durable, au moins dans son volet écologique.

Pour bien des aspects, ces recherches se situent à l'interface entre les Sciences de la Vie, les Sciences de I'Univers et les Sciences de l'Homme et de la Société. C'est particulièrement vrai pour l'étude des conséquences de l'augmentation de la température et du $\mathrm{CO}_{2}$ atmosphérique sur le fonctionnement des écosystèmes, en particulier l'évolution de la matière organique, la croissance des plantes, le rôle des consommateurs herbivores, mais aussi sur les communautés d'arthropodes hématophages dont il est question ici.

Les grandes institutions françaises qui travaillent déjà sur cette thématique préparent ensemble une réponse commune au prochain programme européen ( $6^{\mathrm{e}}$ PCRD) sous la forme d'un projet intitulé «Impact du changement global et des transformations des écosystèmes sur l'émergence et la ré-émergence des maladies à transmission vectorielle ou à hôtes intermédiaires ».

Le Service des Études Médicales d'EDF/GDF a organisé en novembre 2001 un colloque important sur ce thème à Nice. L'ensemble des communications présentées constitue un document de travail particulièrement utile pour les discussions futures sur ce thème (EDF-GDF, 2001). Et cela intervient après toute une série de rapports et de conférences internationales sur le changement climatique (Houghton, 2001 ; EID-Med, 2001).

Le réchauffement de la planète a donné lieu à de nombreux articles de presse ou émissions radio-télévisées. Récemment un article du journal Le Monde prenait en compte les insectes 
(Le Hir, 2002) mais ne disait rien sur les vecteurs. Les observations continues des entomologistes amateurs et professionnels depuis de nombreuses années ont déjà permis de mettre en évidence l'influence des changements climatiques sur certaines aires de répartition (Hughes, 2000 ; Goffart et al., 2001).

\section{Les vecteurs : animaux nuisibles et/ou hématophages. ..}

La grande majorité des vecteurs potentiels sont des insectes mais on ne doit pas oublier d'autres arthropodes hématophages comme les tiques, classées parmi les Acariens.

Certains insectes nuisibles ne sont pas hématophages. Ils ne piquent ni l'homme ni les animaux, mais ils peuvent néanmoins véhiculer des pathogènes sur leurs pattes ou leurs corps en faisant la navette entre les tas de détritus ou de déjections et nos aliments. C'est le cas par exemple des blattes (connues sous le nom de cafards) ou les mouches (mouche verte, mouche de la viande, mouche domestique). Dans le cadre d'un réchauffement climatique, on peut craindre un développement plus rapide des pathogènes et des pullulations de ces insectes. Cela inquiète particulièrement les éleveurs et les agriculteurs qui ont diversifié leurs revenus en développant le tourisme vert «à la ferme ». Les pullulations de mouches, difficiles à contrôler, pourraient dissuader les citadins.

Mais les vecteurs principaux de maladies sont les arthropodes hématophages qui se nourrissent du sang des vertébrés que nous sommes.
Parmi ceux-ci, certains vivent en permanence dans le pelage de leurs hôtes ou bien dans leurs terriers ou leurs maisons. C'est le cas des puces, des poux ou des punaises. Dans cet environnement particulier, ils sont à l'abri des variations climatiques, et l'on peut supposer qu'ils seront peu sensibles aux changements climatiques.

Les autres, essentiellement des diptères et des tiques, vivent une partie ou la totalité de leur cycle dans le milieu extérieur et sont donc sensibles aux variations de climat. C'est particulièrement vrai pour ceux qui ont une phase larvaire et nymphale aquatique et une phase imaginale aérienne, comme les moustiques ou les simulies.

Un bref catalogue de ces vecteurs hématophages en Europe regroupe :

- parmi les diptères : les cératopogonides (par exemple genres Leptoconops ou Culicoïdes, moucherons connus sous le nom d'arabis en Camargue et sur la Côte languedocienne); les simulies; les psychodides ou phlébotomes; les muscides piqueurs (genres Stomoxys, Haematobia ou Haematobosca); les tabanides (ou taons, à la piqûre si douloureuse); les culicidés ou moustiques ;

- parmi les acariens : les tiques.

Pour le reste du monde, il conviendrait d'ajouter à cette liste :

- parmi les diptères : les glossines ou mouches tsé-tsé, vectrices de la maladie du sommeil sur le continent africain ;

- parmi les hétéroptères : les punaises (Réduves), vectrices de la maladie de Chagas sur le continent américain. 
La phrase suivante de Paul Epstein parue en août 2000 a eu un fort retentissement : "Une terre plus chaude sera une terre où le paludisme, le choléra et la dengue s'étendront » (Epstein, 2000).

L'auteur y indique l'impact du réchauffement climatique sur des maladies à transmission directe comme le choléra et sur des maladies à transmission vectorielle comme le paludisme ou la dengue.

Notons que l'impact du réchauffement climatique sur la santé en général a été pris en compte par d'autres auteurs (Besancenot, 2001).

\section{Transmission biologique, transmis- sion mécanique...}

II y a transmission vectorielle lorsque l'agent pathogène, virus, bactérie, protozoaire ou helminthe, est transmis d'un hôte parasité à un hôte sain en passant par l'intermédiaire d'un vecteur qui acquiert le pathogène à l'occasion d'un repas de sang.

Mais cette transmission peut être simplement mécanique si le pathogène ne subit aucune transformation dans le vecteur. Celui-ci joue seulement le rôle d'une seringue volante. Ce sont ses pièces buccales, souillées à l'occasion d'un repas de sang interrompu sur un hôte infecté, qui vont transférer le pathogène à l'hôte sain. Pour cela le délai entre les deux hôtes doit être, en général, très court, de manière à ce que le pathogène reste viable. Dans l'ensemble cela nécessite des conditions bien particulières: une grande concentration d'hôtes potentiels et de vecteurs dans les mêmes lieux. Par exemple, autour des mares résiduelles en saison sèche, et c'est ainsi que l'on peut expliquer la transmission aux bovins des trypanosomes par les taons en Guyane (Raymond, 1989 ; Raymond, 1990).

La plupart du temps la transmission est dite biologique. L'agent pathogène subit un cycle à l'intérieur du vecteur, au cours duquel il peut passer par des stades de multiplication, des stades de schizogonie (reproduction asexuée) ou de sporogonie (reproduction sexuée). Finalement, le pathogène se retrouve dans un stade infectant ou métacyclique, prêt à infecter un hôte sain au prochain repas sanguin du vecteur. On peut citer ici le cycle du Plasmodium, agent étiologique du paludisme, chez son vecteur l'anophèle. Ou le cycle du trypanosome chez la glossine ou mouche tsé-tsé, ou encore celui des filaires chez les simulies, les taons ou les moustiques (Rodhain et al., 1985).

\section{Effets du réchauffement climatique sur les maladies à transmission vectorielle...}

On peut supposer que l'impact du réchauffement climatique sur les maladies à transmission vectorielle pourrait se décliner en (Rodhain, 2000) :

- une augmentation des aires de distribution des vecteurs et des foyers de maladies (en latitude et en altitude);

- une augmentation des périodes d'activités des vecteurs au cours de l'année;

- une augmentation possible de la densité des vecteurs jusqu'à une température optimum, au delà de laquelle l'effet inverse pourrait être observé ;

- une augmentation de la longévité des vecteurs; 
- une diminution de la durée d'incubation des agents infectieux.

Si la plupart des maladies à transmission vectorielle se rencontrent essentiellement en zones intertropicales, les effets indiqués ci-dessus vont bien dans le sens d'une émergence ou d'une ré-émergence de ces maladies dans les zones méditerranéennes et tempérées.

\section{Quelques exemples...}

1) Installation en France d'Aedes albopictus (Skuse, 1894).

Ce moustique, originaire d'Asie du Sud-Est et de l'océan Indien, où il est connu sous le nom de «moustique tigre ", se propage dans le monde à la faveur des échanges commerciaux. En Europe, il a été détecté dès 1979 en Albanie, puis en 1990 en Italie (Schaffner et al., 2000a; Schaffner et al., 2000b).

La femelle, qui pond habituellement ses œufs dans les creux d'arbres, s'est adaptée au monde moderne et pond sur les parois internes des pneus où subsistent souvent des collections d'eau avec de la matière organique. Or ces pneumatiques usagés sont transportés en grande quantité à travers le monde.

C'est ainsi que ce moustique a été découvert pour la première fois en France à l'automne 1999 sur deux sites principaux d'entreposage de pneus usagés : un site dans l'Orne et un dans la Vienne. Des larves, des nymphes et des adultes ont été récoltés. Pour les auteurs de cette découverte (Schaffner et al., 2000a, 2000b), la densité de la population et la multiplicité des gîtes larvaires indiquent une reproduction de l'espèce in situ depuis plusieurs mois, peut-être plusieurs années.

Or ce moustique est vecteur de la dengue (arbovirose) dans sa zone d'origine. En Europe, outre la nuisance du fait de la piqûre, il représente un risque de transmission de virus (West Nile, Sindbis, Tahyna) ou de filaires (Dirofilaria) à l'homme ou aux animaux. Devant ce risque sanitaire, un Comité de surveillance a été mis en place dans notre pays associant médecins, épidémiologistes et entomologistes (Schaffner et al., 2000a).

\section{2) Phlébotomes et leishmanioses}

Dans le sud de la France, les foyers historiques des Cévennes et de Provence ont fusionné en une large zone triangulaire comprise entre Andorre, Nice et Lyon, où des foyers de leishmaniose canine sont observés régulièrement. C'est dans ces foyers que se fait la contamination humaine et que peuvent apparaître des cas de leishmaniose viscérale (Bassenne et al., 1996; Marty et al., 1994). Du fait des échanges fréquents entre le reste du pays et cette région méridionale, notamment à l'occasion des vacances d'été, des foyers ectopiques de leishmaniose canine sont observés plus ou moins régulièrement jusque dans le nord du pays. Les protozoaires parasites du genre Leishmania, à l'origine de ces parasitoses, sont transmis par plusieurs espèces de diptères piqueurs de très petite taille, les phlébotomes.

Des collègues britanniques et italiens ont constitué une vaste base de données concernant les leishmanioses viscérale et cutanée (maladies parasitaires à protozoaires ou hémoprotozooses) dans le bassin méditerranéen 
et plus particulièrement en Italie. Ils se sont rendus compte que les leishmanioses à Leishmania infantum (certaines souches donnent la leishmaniose viscérale, d'autres la leishmaniose cutanée) ne sont présentes que dans l'espace compris entre les isothermes $5-10{ }^{\circ} \mathrm{C}$ en janvier et $20-30{ }^{\circ} \mathrm{C}$ en été. Cela suggère que la température est l'un des facteurs principaux limitant la diffusion de ces maladies vers le nord de l'Europe. Et il se pourrait bien que d'ici à 30 ans, la Grande Bretagne ait un climat assez chaud pour supporter le développement à la fois des leishmanies et de leurs vecteurs, les phlébotomes (Kuhn, 1999).

L'attention a été portée récemment en Italie sur l'augmentation importante de l'incidence de la leishmaniose viscérale, et ceci en dehors de tout lien avec le virus HIV ou d'autres immunosuppressions. L'examen de la base de données a montré que la présence des vecteurs était déterminée principalement par les températures minimums en été et en hiver. Et les deux vecteurs impliqués Phlebotomus perniciosus et $P$. perfiliewi ont des exigences écologiques différentes, expliquant leurs distributions complémentaires. Nos collègues ont fait varier les paramètres de température dans leur modèle pour simuler ce qu'il pourrait advenir dans le futur. Le modèle prévoit clairement une augmentation très nette de l'abondance de l'un des vecteurs $P$. perfiliewi en Italie, qui pourrait aller en Europe du Nord jusqu'à la latitude $49^{\circ} \mathrm{N}$. L'autre vecteur pourrait se développer plus facilement en altitude et devenir ainsi plus abondant en Suisse par exemple. Concernant les deux formes de leishmaniose, les modèles prédisent une augmentation très importante de la leishmaniose viscérale en Europe (Kuhn, 1999).

Lors de la conférence de Nice, nos collègues italiens ont indiqué qu'ils avaient trouvé de nouveaux foyers de leishmaniose canine dans les régions de Vérone et de Turin, et qu'ils avaient trouvé Phlebotomus perniciosus en Val d'Aoste en 2001, dans des stations qui étaient négatives dans les années 60 (Ferroglio et al., 2001).

Toujours lors du colloque de Nice, les leishmanioses ont été placées en première priorité parmi les maladies à transmission vectorielle à prendre en compte dans le cadre des changements climatiques. Ceci en raison de leur importance à la fois vétérinaire et médicale. L'attention a notamment été attirée sur le problème d'apparition possible de cas de résistance des parasites aux médicaments actuellement disponibles, en raison d'une mauvaise utilisation des produits vétérinaires (durée et coût élevé du traitement, entraînant des interruptions).

\section{3) Moustiques et fièvre à West Nile}

Parmi l'ensemble des maladies à vecteurs, les arboviroses tropicales sont sans doute les plus à craindre de l'impact des modifications climatiques. Les épidémies récentes, et toujours d'actualité, à virus West Nile aux ÉtatsUnis, en Israël et en France témoignent de cette situation (Duvallet, 2001).

Le cycle de transmission classique du virus West Nile implique des oiseaux et des moustiques. Certaines espèces de moustiques hébergent et amplifient le virus, jouant ainsi à la fois le rôle de vecteur et d'hôte. Les moustiques infectés transmettent le virus à d'autres oiseaux lors d'un repas de sang. Les oiseaux infectés peuvent 
transmettre ensuite le virus à d'autres moustiques. Certains oiseaux sont malades et meurent alors que d'autres s'infectent sans montrer de signes cliniques. Mais à l'occasion d'un repas de sang pris sur mammifère, les moustiques peuvent aussi leur transmettre le virus. Cependant la virémie chez les mammifères est généralement considérée trop faible pour permettre l'infection d'autres moustiques. Ces mammifères, en particulier l'homme et le cheval, sont considérés comme des « culs-de-sac » épidémiologiques.

Les oiseaux sauvages sont donc les réservoirs essentiels de ce virus qui peut entraîner une évolution fatale chez un certain nombre d'individus. Cette sensibilité des oiseaux à la maladie les fait utiliser comme sentinelles pour surveiller l'évolution de l'épizootie.

Depuis l'apparition du virus West Nile pour la première fois sur le continent américain en 1999, un système de surveillance extrêmement actif a été mis en place dans les zones concernées pour les hommes, les animaux (en particulier oiseaux et chevaux) et les moustiques (Nash, 2000).

En 1999, 59 cas humains d'encéphalite incluant 7 décès ont été observés. En 2000, 21 cas humains d'encéphalite incluant 2 décès et en 2001 au moins 42 cas humains (dont 2 décès), et plus de 170 chevaux, 5710 oiseaux sauvages, 736 lots de moustiques ont été découverts positifs.

Au cours de cette épidémie américaine, certaines espèces d'oiseaux ont payé un lourd tribut en terme de mortalité, et des anticorps ont aussi été trouvés chez 26 espèces de mammifères (incluant chevaux, lapins, écureuils, chauve-souris et chats). Plus de 10 espèces différentes de moustiques ont été impliquées. Pendant l'été 2001, le virus s'est répandu vers le Sud (Floride), vers le Nord (il est entré en août au Canada) et vers l'Ouest (Indiana).

Pour la France, en 1962, 80 chevaux furent atteints en Camargue dont 25 à $30 \%$ sont morts, la plupart euthanasiés. Le virus a été isolé en 1964 de Culex modestus et du sang de 2 entomologistes travaillant dans la zone. Pour les hommes, 13 cas, dont un mortel, ont été identifiés. Le virus a de nouveau été isolé de Culex modestus et d'un cheval en 1965 (Hannoun et al., 1969). Une enquête sérologique, menée de 1975 à 1979, a révélé des prévalences de $4,9 \%$ dans la population humaine et de $2 \%$ chez les chevaux.

En 2000, 76 chevaux ont montré des signes cliniques et des sérologies positives contre West Nile en Petite Camargue. Une vingtaine de chevaux sont morts, certains euthanasiés. Aucun cas humain n'a été détecté dans cet épisode.

Une vaste enquête pluridisciplinaire de surveillance épidémiologique a été mise en place dans cette zone (Murgue et al., 2000 ; Zientara et al., 2001).

\section{4) Culicoïdes et Blue Tongue}

C'est une arbovirose transmise par de petits diptères de la famille des Cératopogonides. Le principal vecteur en Afrique est Culicoides imicola, dont la limite de répartition était classiquement décrite au niveau du $40^{\circ}$ parallèle $\mathrm{N}$. Mais durant ces dernières années, et de manière progressive, il a été signalé en Grèce continentale (1998), en Bulgarie (1999), en Espagne, en Italie et, pour la première fois, en Corse 
en septembre 2000. Son apparition a été accompagnée d'épidémies très sévères de fièvre catarrhale dans les cheptels ovins (Zientara et al., 2000).

Les conditions climatiques favorables de l'hiver 2000/2001 n'ont pas permis d'éliminer cet insecte tropical, maintenant bien installé sur l'île de beauté. Son abondance a été à l'origine de la résurgence de la maladie assez tôt durant l'été 2001.

Le continent est désormais sous la menace directe d'une extension de la distribution du vecteur $C$. imicola. Les conséquences de l'apparition de foyers seraient en effet très lourdes (Zientara et al., 2000).

Cette fièvre catarrhale du mouton peut être considérée comme un modèle de maladie vectorielle émergente menaçant le continent européen (De La Rocque et al., 2001).

\section{5) Tiques et maladies transmises}

Plus de 800 espèces de tiques sont connues dans le monde. Leur cycle de développement peut comprendre de 1 à 3 hôtes différents. Ce sont les vecteurs potentiels de nombreux pathogènes : spirochètes et rickettsies, arbovirus, protozoaires (Rodhain et al., 1985). Parmi les maladies transmises, les plus importantes en Europe en raison de leur prévalence et de l'augmentation de leur incidence sont la maladie de Lyme (ou borréliose à Borrelia burgdorferi s.l.) et les encéphalites virales, qui ont un vecteur commun Ixodes ricinus.

La maladie de Lyme est présente dans toute la zone de distribution de la tique, alors que les encéphalites ne sont présentes qu'en quelques foyers bien délimités. Nos collègues britanniques (Randolph, 2001) ont étudié l'impact du réchauffement climatique sur ces maladies. Dans le cas des encéphalites, les foyers semblent directement sous la dépendance des conditions climatiques, et les modèles d'évolution des climats montrent clairement un déplacement de ces foyers vers le nord de l'Europe en direction des pays scandinaves. Les observations actuelles sur le terrain (diminution du nombre de cas dans le sud de la zone de distribution et augmentation en Suède) confirment ces prédictions.

Par contre, la maladie de Lyme dépend aussi fortement de conditions biotiques comme la présence et l'abondance des hôtes de la tique (Cervidés) et l'on ne peut expliquer l'évolution épidémiologique de la maladie en utilisant uniquement des modèles climatologiques. Rappelons aussi que les activités humaines et les modifications des écosystèmes (urbanisation, loisirs) vont dans le sens d'une augmentation du contact entre les tiques et les humains.

\section{Conclusion}

Les activités humaines modifient considérablement et rapidement la biosphère et l'atmosphère au point de perturber le fonctionnement des sociétés humaines, de mettre en péril de nombreuses espèces et de favoriser le développement d'espèces nuisibles. Les changements globaux relatifs à la biodiversité et au climat ont maintenant largement débordé la sphère des milieux scientifiques et font l'objet de conventions internationales (Rio, Kyoto), généralement difficiles à mettre en œuvre (conférence de La Haye, Bonn...). Les conflits d'intérêts sont nombreux et les connaissances 
scientifiques encore trop souvent insuffisantes pour en faciliter l'arbitrage et les prises de décision politiques.

Même s'il est difficile d'écrire l'histoire du futur, on peut, sans risque de se tromper, prédire des modifications dans l'aire de répartition des vecteurs, des modifications dans la distribution des foyers de maladie, donc des risques nouveaux à prendre en compte.

Il ne s'agit pas pour nous de lancer un cri d'alarme, mais d'alerter nos concitoyens et les responsables politiques, car des recherches en écologie et en entomologie médicale sont indispensables pour mieux comprendre l'épidémiologie changeante de ces maladies. Des systèmes de surveillance pérennes des vecteurs et des maladies doivent être mis en place, en particulier sur la rive sud de l'Europe et tout autour de la Méditerranée, pour déceler suffisamment tôt des risques potentiels. Or, les formations universitaires de haut niveau en entomologie générale et en entomologie médicale ont pratiquement disparu dans notre pays, et pour certains groupes de vecteurs, nous n'avons plus de spécialistes pour les identifier.

\section{RÉFÉRENCES BIBLIOGRAPHIQUES}

Bassenne I., Pratlong F., Dereure J., Balard Y., Dedet J.P., 1996. La leishmaniose humaine en Cévennes : étude rétrospective 1933-1994. Med. Mal. Infect., 26: 1164-8.

Besancenot J.P., 2001. Climat et santé. Coll. Médecine et Société, Paris, PUF, $128 \mathrm{p}$.

De La Rocque S., Hendrikx P., 2001. Impact du changement climatique sur la santé : exemple de la fièvre catarrhale du mouton ou "blue tongue". Colloque Changement climatique et maladies à vecteurs, Nice, $18-20$ novembre 2001, SEM, EDF-GDF, Paris.

Duvallet G., 2001. Epidémiologie et manifestations cliniques de l'infection à virus West Nile. Colloque Changement climatique et maladies à vecteurs, Nice, 18-20 novembre 2001, SEM, EDFGDF, Paris.

EDF-GDF, 2001. Changement climatique et maladies à vecteurs. Colloque Nice, 18-20 novembre 2001, Service des Etudes médicales, Paris, France.

EID-Med (Entente interdépartementale pour la démoustication-Méditerranée), 2001. Changement climatique. Fantasme ou réalité. Actes du Colloque, 15 mai 2001, Montpellier, France.

Epstein P.R., 2000. Is global warming harmful to health? Sci. Am., August:5057.

Epstein P.R., 2001. Climate change and emerging infectious diseases. Microbes and Infection, 3: 747-754.

Ferroglio E., Rossi L., Mignone W., Maroli M., Raimondo C., Gastaldo S., Trisciuoglio A., 2001. Preliminary reports about endemic canine leishmaniasis foci in North-west Italy. Colloque Changement climatique et maladies à vecteurs, Nice, 18-20 novembre 2001, SEM, EDF-GDF, Paris.

Goffart P., de Schaetzen R., 2001. Des libellules méridionales en Wallonie: une conséquence du réchauffement climatique. Forêt wallone, 51: 2-5.

Hannoun C., Panthier R. \& Corniou B. 1969. Epidemiology of West Nile infection in the south of France. in: Bardos V., editor. Arboviruses of the California complex and the Bunya-mwera group. Bratislava: Publ. House SAS. 379-387.

Houghton J.T., Ding Y., Griggs D.J., et al., 2001. Climate Change 2001: The Scientific Basis. Contribution of Working Group । to the Third Assessment Report of the Intergovernmental Panel on 
Climate Change (IPCC). Cambridge: Cambridge University Press, 2001.

Hughes L. 2000. Biological consequences of global warming: is the signal already apparent ? TEE, 15(2): 56-61.

Kuhn K.G., 1999. Global warming and leishmaniasis in Italy. Bull. Trop. Med. Intern. HIth., 7(2) : 1-2.

Le Hir P., 2002. Les insectes aux avantpostes du réchauffement climatique. Le Monde (12-13 mai 2002) : 26.

Marty P., Le Fichoux Y., Pratlong F., GariToussaint M., 1994. Human visceral leishmaniasis in Alpes-Maritimes, France: epidemiological characteristics for the period 1985-1992. Trans. $R$. Soc. Trop. Med. Hyg., 88: 33-34.

Murgue B., Murri S., Zientara S., Durand B., Durand J.P., Zeller H., 2001. West Nile outbreak in horses in Southern France (2000): the return 35 years later. Emerg. Infect. Dis., 7: 692-696.

Nash D. et al., The outbreak of West Nile virus infection in the New York City area in 1999. The New England Journal of Medicine, 344: 1807-1814.

Randolph S.E., 2001. The shifting landscape of tick-borne zoonoses: tickborne encephalitis and Lyme borreliosis in Europe. Philos. Trans. R. Soc. Lond. B, Biol. Sci., 356: 1045-56.

Raymond H.L., 1989. Distribution temporelle des principales espèces de taons nuisibles au bétail en Guyane française. Ann. Soc. Ent. Fr., 25(3) : 289-294.
Raymond H.L., 1990. Tabanus impotunus, vecteur mécanique expérimental de Trypanosoma vivax en Guyane française. Ann. Parasitol. Hum. Comp., 65(1) : 44-46.

Rodhain F. et Perez C., 1985. Précis d'entomologie médicale et vétérinaire. Maloine, Paris, $458 \mathrm{p}$.

Rodhain F., 2000. Modifications climatiques : impacts potentiels sur la santé. L'exemple des maladies à vecteurs. Bull. Ass. Anc. Elèves Inst. Pasteur, 162: 8-14.

Schaffner F., Karch S., 2000a. Première observation d'Aedes albopictus (Skuse, 1894) en France métropolitaine. C. $R$. Acad. Sci. Paris, Sciences de la vie, 323: 373-375.

Schaffner F., Delaunay P., 2000b. Aedes albopictus (Skuse, 1894) (Diptera: Culicidae) établi en France métropolitaine. Congrès Société française de Parasitologie, 1-3 mars 2000, Montpellier, France.

Zientara S., de La Rocque S., Gourreau J.M., Grégory M., Diallo A., Hendriks P., Libeau G., Sailleau C., Delécolle J.C., 2000. La fièvre catarrhale ovine en Corse en 2000. Epidémiol. et santé anim., 38 : 133-144.

Zientara S., Moutou F., Durand B., Dufour B. et Plateau E., 2001. L'encéphalite à virus "West Nile": situation et évolution en France 2000-2001. Bull. Soc. Vét. Prat. de France, 85: 44-50. 